\title{
LA LAICITÉ IN QUESTIONE. UN COMMENTO AD UN TESTO DI LUCA
}

DIOTALLEVI

Ari Pedro Oro ${ }^{1}$

Claude Petrognani ${ }^{2}$

Il tema della laicità, e della secolarizzazione, suo equivalente più vicino, costituiscono oggetti di controversia e polemica nelle Scienze Sociali. Questo perché, come segnala Jeffrey Hadden (1987), più che teorie, quei concetti rappresentano una dottrina e anche un'ideologia, o, come sostiene Pierre Sanchis, si può paralare, a proposito di laicità e secolarizzazione, di un progetto e, addirittura, di desideri personali. Così si espressa quest'ultimo autore: "Forse non c'è nelle Scienze Sociali altro campo in cui gli studiosi investano con più intensità i desideri frutto di storie di vita conflittuali - nei sensi, oltretutto, i più inaspettati" (Sanchis, 2001, p. 31).

Ł̀ evidente che niente di tutto ciò si applica direttamente al testo di Diotallevi qui commentato, il quale è molto prudente nelle sue affermazioni, acuto nelle sue analisi e perspicace nelle sue considerazioni finali. Oltretutto, Diotallevi dimostra di possedere un'ampia padronanza per quanto riguarda il tema della laicità e delle teorie al riguardo. Per farsene un'idea, è sufficiente vedere l'ampia bibliografia al riguardo per scrivere questo testo, il quale, in questo senso, è un contributo importante per il pubblico di lingua portoghese che desidera avvicinarsi al tema qui proposto da Diotallevi, e che, spesso, incontra difficoltà per accedere all'ampia letteratura internazionale esistente.

Nel suo testo sul "separatismo moderato" Diotallevi si occupa della religione nello spazio pubblico analizzando due modelli di separatismo, il francese della laicità e quello statunitense della libertà religiosa. In entrambi, sono analizzate le relazioni vigenti tra potere politico, religioso e giuridico. Dunque, la sua analisi si avvale della teoria di Luhmann come supporto per comprendere l'attualità delle relazioni tra questi poteri.

Il nostro commento al testo di Diotallevi si focalizza su un'unica questione: la laïcité francese. Lungi dal voler polemizzare con l'autore, vorremmo, tutt'al più, procedere relativizzando e introducendo alcune questioni e, eventualmente, avanzando alcune affermazioni su questo tema. Ci siamo avvalsi, per le nostre considerazioni, sulle analisi realizzate dal sociologo francese Jean Baubérot, autore di diversi testi sulla laicità. Procedendo in tal senso, siamo coscienti, però, del peso che riveste, sulle nostre considerazioni, l'unilateralità del nostro approccio e della nostra

1Professor titular do Departamento de Antropologia e do Programa de Pós-Graduação em Antropologia Social da Universidade Federal do Rio Grande do Sul.

2Doutorando do Programa de Pós-Graduação em Antropologia Social da Universidade Federal do Rio Grande do Sul. Objeto da tese: as relações entre futebol e religião. 
prospettiva, nonostante ci si appropri delle idee di uno dei più grandi specialisti francesi sul tema della laicità ${ }^{3}$.

\section{La laicità francese secondo Baubérot}

Scrive Luca Diotallevi a proposito della laïcité: “nell'ambito del separatismo radicale, la laïcité finisce per rappresentare una eccezione nella eccezione, ma anche una eccezione in crisi. Ciò è del resto quanto concordemente testimoniano i suoi critici ed i suoi difensori” (Diotallevi, 2014, p.3) eccetto, come chiarisce in una nota a piè di pagina "posizioni estreme ed isolate" (Diotallevi, 2014, p. 4) riferendosi al testo di Jean Baubérot Le tante laicità nel mondo (2008)traduzione dal francese del libro Les laïcités dans le monde (2007).

Infatti, in questo testo, così come in tanti altri, Baubérot, considerato una referenza incontournable sul tema della laïcité ${ }^{4}$, sostiene che laïcité n'est pas une exception française" (Baubérot, 2007, p.119), rifacendosi a fatti storici precisi e incontestabili.

Per quanto riguarda la crisi della lä̈cité francese, ammesso che ci sia, sarebbe opportuno domandarsi fino a che punto possa decorrere, come suggerisce Diotallevi, dal fatto che nella lä̈cité lo spazio pubblico risulta "omogeneo e dominato dal potere politico (...) collocando la religione nel privato" (Diotallevi, p.5), mentre per quanto riguarda il caso del religious freedom lo spazio pubblico risulta, al contrario, "disomogeneo e sottratto ad ogni egemonia (...) garantendo uno spazio pubblico alla religione" (p.5).

Ora, a Parigi (come a New York), lo spazio pubblico risulta eterogeneo: moschee, templi, chiese, cattedrali, sinagoghe si trovano sparpagliate per la città: sarebbe sufficiente, per esempio, passeggiare per il quartiere Le Marais, nella capitale francese, per rendersi conto degli innumerevoli ristorantini e take away di cucina ebraica servendo i falafel e persone deambulando indossando la kippah. E che dire della meravigliosa moschea situata sulla rive gauche, vicina al famoso Quartier Latin, e delle numerose signore indossando la hijab?

3Elenchiamo alcuni libri de Jean Baubérot sul tema della laicità: Laicité 1905-2005, entre passion et raison. Paris, Seuil, 2004; Les laïcités dans le monde. Paris, PUF, 2007 ; La laïcité falsifiée. Paris, La découverte, 2012; Histoire de la laïcité en France, PUF, Paris, 2010; Baubérot, J.- Milot, M. Laïcités sans frontières. Paris, Seuil, 2011

4È opportuno ricordare che per questo sociologo francese, la laicità "implique la dissociation du champ politique (comme instance de pouvoir) et du champ religieux (comme instance d'autorité), l'autonomie du politique dans la régulation normative d'une société pluraliste, notamment au plan juridique” (Baubérot, 2010, p. 621). 


\section{LA LAICITÉ IN QUESTIONE. UN COMMENTO AD UN TESTO DI LUCA}

DIOTALLEVI 3

Infatti, come vedremo in un secondo momento, sempre seguendo le analisi di Baubérot, la laïcité francese, così come è espressa dalla legge di separazione del 1905, legge che Baubérot considera come la "regola d'oro della laicità francese" (Baubérot, 2011, p.63) non prevede e non attua il dominio dello Stato nello spazio pubblico, relegando (come sembra sostenere Diotallevi) la religione nella sfera del privato.

Il fondamento della separazione Chiesa/Stato in Francia (così come negli Stati Uniti) riposa sulla costruzione di una società civile nella quale le persone di diverso credo religioso e differente estrazione culturale e politica possano convivere pacificamente, una società laica nella quale lo Stato garantisce le stesse libertà e gli stessi diritti a chiunque. Così, la laïcité "est un concept irremplaçable ${ }^{5}$ " (Baubérot, 2011)che non limita le libertà religiose, ma, al contrario, le sostenta e le amplia: " la libertà laica (è) attenta non soltanto alla libertà di religione, ma anche alla libertà dalla religione” (Baubérot, 2011, p.59).

Secondo Baubérot, quando la politica, in nome della "sacralità" della laïcité, approva leggi restrittive come quella che proibisce agli alunni di esibire segni ostentatori di appartenenza religiosa nelle scuole (2004), o le donne di indossare in pubblico il burqa (2010), (la politica) sta facendo una interpretazione illegittima della legge del 1905, falsificandola (Baubérot, 2012), stigmatizzando, così, per fini politici, in particolare una religione, l'Islamismo.

\section{1) La "laïcité", un'eccezione francese?}

Questa formula: "La laïcité est une exception française", adottata in Francia nel finale degli anni 1980 (Baubérot ricorda che fu nel 1989, in occasione di un seminario intitolato "la laïcité, une exception à la française" che quest'espressione fu adottata per la prima volta) è diventata così evidente e di dominio comune, per i francesi ma non solo, che, come scrive ironicamente il sociologo francese " a fait écrire des choses fausses à des personnes prétendant avoir autorité en la matière” (Baubérot, 2007, p. 119). Esempio: nel 2007, l' Haut Conseil à l'intégration si pronuncia così : "objet d'étonnement pour tout le monde, la loi de séparation (de 1905) a suscité des émules

5Scrive Baubérot (2011): “ les laïcités ont toutes en commun le fait d'articuler quatre principes (...) la garantie de la liberté de conscience, l'égalité et la non-discrimination, la séparation du politique et du religieux, la neutralité de l'État à l'égard des divers croyances. Le terme laïcité est donc irremplaçable." Disponível em: jeanbauberotlaicite.blogspirit.com 
et fait naître de imitations" (Baubérot, 2011, p.120), indicando il caso del Messico. Questo è un falso perché in Messico la legge di separazione fu stabilita nel 1861 e accentuata nel 1874. Dunque, non risulta che sia stato il Messico ad imitare la Francia, ma bensì il contrario, "loin d'avoir imité la France, le Mexique a constitué une référence dans l'élaboration de la loi française de séparation des Eglises et de l'État" (Baubérot, 2011, p.120), come d'altronde è documentato nella relazione della"Commission parlementaire", redatta da Aristide Briand, il quale cita lungamente la legge di separazione messicana del 1874: “Le Mexique possède ainsi la législation laïque la plus complète qui ait jamais été mise en vigueur jusqu'à a ce jour. Il est délivré depuis trente ans de la question cléricale et a pu se vouer entièrement à son développement économique: il connaît réellement la paix religieuse" (In: Baubérot, 2011, p.120).

Inoltre, Briand ricorda che la Francia, adottando la legge di separazione del 1905, non ha fatto altro che aggiungersi ad un gruppo di paesi che in precedenza avevano adottato la legge di separazione Chiesa/Stato, tra cui: il Canada, gli Stati Uniti, il Brasile e il Messico (In: Baubérot, 2011, p.120).

Dunque, introducendo la separazione, nel 1905, tra Chiesa/Stato, la Francia "ne fait donc que rejoindre ce troisième groupe où plusieurs pays l'ont déjà précédée. On est à l'oppose d'une vision de la laïcité exceptionnaliste" (Baubérot, 2011, p.120).

Quindi, sarebbe opportuno domandarsi fino a che punto, come sembra invece dire Diotallevi, ci si possa riferire alla laicità come una "eccezione francese".

\section{2) La "Laïcité", dominio dello Stato nello spazio pubblico ?}

Nell'articolo Libertà religiosa e laicità francese, Baubérot (2011) dopo aver realizzato una rapida ricostruzione storica della laïcité francese, presenta e commenta la famosa legge del 1905.

La presenta come una "regola d'oro", come abbiamo già menzionato in precedenza, considerandola il fondamento giuridico principale della laicità francese (Bauérot,2011, p.64).

Procedendo ad una rapida analisi degli articoli e emendamenti principali costitutivi della legge del 1905 l'autore percepisce che non si trattava di una restrizione/esclusione delle religioni nello spazio pubblico, ma, al contrario, la stessa legge ha permesso alle minoranze religiose di acquisire maggiore visibilità.

Infatti, come sottolinea Baubérot (2011, p.64) "aspetti di questa legge mostrano che essa è politicamente liberale e accomodante dal punto di vista religioso”. Gli articoli XII e XVII della 


\section{LA LAICITÉ IN QUESTIONE. UN COMMENTO AD UN TESTO DI LUCA}

DIOTALLEVI 5

legge del 1905, che regolano la distribuzione degli edifici religiosi (proprietà pubblica dopo la Rivoluzione Francese, o costruiti nel secolo XIX con finanziamento pubblico ${ }^{6}$ ) concedono gratuitamente questi spazi di culto (un emendamento che voleva introdurre il pagamento di un affitto è stato respinto con 475 voti contro 95); l'articolo II specifica che il potere pubblico può dispensare spese relative a servizi di elemosina e per facilitare il libero esercizio di culto; la proposta di un emendamento per sostituire il calendario delle feste religiose è stato respinto (con 466 voti contro 60), allo stesso modo, un emendamento che voleva rendere obbligatorio la cittadinanza francese per chi volesse diventare ministro di culto fu anch'esso respinto (con 460 voti contro 60); un emendamento teso a proibire l'uso dell'abito talare (tipica veste ecclesiastica) nello spazio pubblico fu respinto (con 391 voti contro 184); fu confermata la libertà di processione ( $d i$ religione e dalla religione) nello spazio pubblico (articolo 31). Infine, come ricorda Baubérot (2011), la legge di separazione del 1905 non fu applicata alle colonie ${ }^{7}$ e neanche alle regioni della Alsazia e Mosella, dove, per ragioni storiche ${ }^{8}$, è rimasto in vigore (fino ad oggi) il concordato del 1801.

Come detto, anche l'Islamismo ha beneficiato della legge del 1905. Ricorda Baubérot che in Francia, oggi, l'Islamismo è la seconda religione, dopo il cattolicesimo, e, malgrado la mancanza di statistiche ufficiali sul numero di musulmani su territorio francese, si può desumere che "la stima del numero dei musulmani presenti nella Francia metropolitana varia da 2,1 milioni (Ined) a 5/6 milioni di musulmani, di cui il 20/ 25\% sarebbero praticanti” (Baubérot, 2011, p. 66).

Negli anni 20 del secolo scorso, dunque quindici anni dopo l'introduzione della legge del 1905, si calcolava che il numero dei musulmani in Francia non superasse le 150.000 persone $^{9}$, ma nonostante ciò, commenta Baubérot (p.65), "una grande moschea fu costruita a Parigi e la sua costruzione beneficiò di sovvenzioni pubbliche".

6A titolo informativo, "la maggior parte delle chiese cattoliche, circa la metà dei templi protestanti e un terzo delle sinagoghe ebraiche” (In: Baubérot, p.64).

7In Algeria, per esempio, sarà mantenuta una politica gallicana, nonostante le rivendicazioni di alcuni algerini affinché l'Islamismo potesse beneficiare della legge del 1905 (Bozzo, 2006. In: Baubérot, 2011, p.65).

8Si sa che i dipartimenti dell'Alsazia e della Mosella, che nel 1871 erano diventati tedeschi, ritornarono sotto dominio francese nel 1919, e che la legge di laicizzazione della scuola pubblica (la legge Ferry del 1882) così come la legge di separazione Chiesa/Stato, del 1905, non furono applicate (In: Baubérot, 2011, p.65):

9Le cifre sono disponibili sul sito www.wikipedia.org/wiki/Islam en France. 
Coloro che protestarono in nome della legge del 1905, perché violerebbe l'articolo II, che afferma che "La Repubblica non riconosce, né stipendia e sovvenziona alcun culto" (In: Baubérot, 2011, p.63), fu risposto che la decisione di sovvenzionare la costruzione della moschea applicava semplicemente il principio dell'uguaglianza tra diverse religioni, dispositivo costante della legge del 1905. A questo proposito scrive Baubérot (2011, p.65) che "Edouard Herriot, personalità laica, considerato molto rigido", dichiarò: "non c'è alcun impedimento a dare ai musulmani una moschea dal momento che legittimamente diamo ai cattolici chiese, ai protestanti templi e agli ebrei sinagoghe” (Poulat, 2003, p. 185 In: Baubérot, 2011, p.65).

Questa interpretazione della legge del 1905 ha continuato a prevalere e, nonostante le numerose contestazioni e tentativi per ostacolare la costruzione di moschee, il suo numero è aumentato accompagnando così l'aumento della popolazione musulmana. Baubérot (2011, p.66) specifica che oggi la Francia possiede circa 90 moschee e che più di un centinaio sono, attualmente, in costruzione, e più di 2.368 sale di preghiera.

Il Consiglio di Stato, il 19 luglio del 2011, ha proposto, per mediare tra questi due aspetti della legge del 1905 (articolo II) - quello che impedisce al potere pubblico di finanziare i culti, e quello che garantisce il diritto di praticarli- la costruzione di "sale polivalenti, una delle quali serva alle cerimonie del culto musulmano a condizione che non sia né esclusiva, né perpetua" (Baubérot, 2011, p.66).

Inoltre, sempre il Consiglio di Stato ha stabilito che i sussidi pubblici non violano l'articolo II: infatti, è fissata una distinzione tra il cultuale (che non può essere sussidiato) e il culturale (che può essere sussidiato). Questo significa che i sussidi pubblici, posti qua in gioco, servano per il bene comune (culturale) e non per un culto particolare (cultuale): "il Consiglio di Stato autorizza sovvenzioni pubbliche per la costruzione di un ascensore in una chiesa cattolica, invocando uno interesse pubblico locale (...) parimenti autorizza il finanziamento di un macello per la carne hallal, per ragioni di igiene e salute pubblica”. (Baubérot, 2011, p.67). Si tratta, dunque, di una applicazione moderata della legge del 1905 che è conforme alla giurisprudenza (Baubérot, 2011, p.66) $)^{10}$.

10 Il Consiglio di Stato ha ricordato, anche, che dopo la legge del 1905 sono state costruite, secondo questo iter, circa di 450 chiese cattoliche. 


\section{LA LAICITÉ IN QUESTIONE. UN COMMENTO AD UN TESTO DI LUCA}

DIOTALLEVI 7

A partire da queste considerazioni non sembrerebbe esserci, dunque, nella legge del 1905, qualcosa che possa indicarci che la separazione Chiesa/Stato fu realizzata in Francia per escludere le religioni nello spazio pubblico.

Inoltre, è bene ricordarlo, che il campo dell'educazione in Francia è anch'esso eterogeneo: ossia lo Stato, anche per quello che concerne il campo dell'educazione, non è detentore del dominio nello spazio pubblico. Questo significa che a lato delle scuole pubbliche e laiche (la legge di laicizzazione della scuola pubblica di J. Ferry è datata 1882) si trovano scuole confessionali (cattoliche, ebraiche e musulmane) le quali, senza ricevere sussidi dallo Stato ${ }^{11}$, possono esercitare l'insegnamento conforme il proprio credo religioso. Baubérot ricorda che l'introduzione della legge del 2004 (che analizzeremo successivamente) ha prodotto un aumento del numero di giovani ebrei nelle scuole confessionali ebraiche che utilizzano il copricapo kippah (Baubérot, 2011, p. 68). Questa disposizione (2004) ha prodotto anche un aumento del numero delle scuole musulmane (Id., Ibid.,).

Dunque, la laïcité, così come la intende Baubérot, non ha prodotto una separazione tra Chiesa/Stato nella quale il potere politico confinerebbe le religioni nella sfera del privato. Ossia, detto in altro modo, non sembra esserci una subordinazione del religioso nei riguardi del politico nello spazio pubblico, tesi questa ricorrente e che Diotallevi sembra sostenere.

Tuttavia, non possiamo negare che la legge votata nel 2004 (detta "legge di laicità", 15/03/2004, e che ha semplificato, come dice Baubérot, (2011, p. 67) "nella visione di alcuni, la laicità a questa sola questione"), che proibisce agli alunni di indossare nella scuola simboli vistosi di appartenenza religiosa, così come la legge del 2010 (imposta in nome "dell' ordine pubblico" e non della "laicità") che proibisce l'uso del foulard integrale (burqa o niqab) nello spazio pubblico, non diano ragione a Diotallevi. Infatti, la legge del 2004 e quella del 2010 instaurano un controllo dello Stato sulle religioni, proibendo e sanzionando certe pratiche religiose.

Baubérot, nel suo libro La laïcité falsifiée (2012), cerca di dimostrare, però, che la laicità, cosi come è stabilita dalle leggi del 2004 e 2010, sia un'impostura, una falsificazione che, al contrario, ferisce lo spirito e i principi della laïcité del 1905.

In un'intervista al giornale La Croix (il 10/02/2012) Baubérot afferma :

"On voit ainsi apparaître ce que j'appelle, à la suite de François Baroin, une "nouvelle laïcité », synonyme d'un contrôle de l'État sur la religion et de répression de ce qui échappe à ce contrôle. Cette laïcité ne ressemble pas à notre "laïcité historique », dont la loi de 1905 a fixé le

11 Nonostante ciò, sappiamo che ci sono scuole private (confessionali o no) che possono ricevere sussidi dallo Stato, a condizione che firmino un contratto. A questo proposito si può consultare il sito www.education.gouv.fr 
cadre. C'est cette imposture historique et symbolique que j'ai voulu démonter. La loi de 1905 pose que la religion n'est pas affaire d'Etat et qu'elle doit être distincte de la puissance publique. Mais cela ne veut pas dire que la religion soit confinée à la "sphère intime». Au contraire, la loi envisage la liberté de conscience et le libre exercice des cultes comme une liberté publique. Elle ne dit pas : "Cachez cette religion que je ne saurais voir»... Les ultralaïques ont toujours voulu imposer cette optique. Ils ont été battus en 1905, mais ils dominent aujourd'hui".

Dunque, si suggerisce che ad una supposta declinazione del concetto di laicità al singolare si sostituiscano molte declinazioni della laicità al plurale:

“Au pluriel, car suivant les traditions nationales et régionales, les conjonctures géo-politiques, les mutations sociales dominantes, les périodes socio-historiques, différents types de lä̈cité s'avèrent plus ou moins hégémonique. La réalité empirique est infinie et mêle des ingrédients multiples" (Baubérot, 2011 In: jeanbauberotlaicite.blogspirit.com).

Per questo motivo, e ritornando sull'affermazione di Diotallevi della laicità francese come una "eccezione nella eccezione", ci sembra proficuo indagare, vista l'eterogeneità e la complessità del concetto di laicità, circa la pertinenza e il valore euristico dei significati altri della laicità che trascendono, in questo modo, il doppio regime di separatismo così ben analizzato da Diotallevi nel testo qui commentato.

\section{Referenze bibliografiche}

BAUBEROT, Jean. La représentation de la laïcité comme "exception française ». In: Cosmopolitique. N. 16 de novembro, 2007.

. Libertà religiosa e laicità in Francia. In: «Lessico di Etica pubblica », 2 (2011), n² ISSN 2039-2206.

. Les laïcités dans le monde, PUF, Paris, 2007.

. La laïcité falsifiée, La découverte, Paris, 2012.

. Histoire de la laïcité en France, PUF, Paris, 2010.

. Laicité/laicisation. In Azria, Régine et Hervieu-Léger, Danièle. Dictionnaire des faits religieux. Paris, PUF, 2010, p. 620-624.

BAUBÉROT, J.- Milot, M. Laïcités sans frontières, Seuil, Paris, 2011.

HADDEN, Jeffrey. Toward desacralizing secularization theory. In: Social Forces, N. 65, 1987, p. 587-611. 


\section{LA LAICITÉ IN QUESTIONE. UN COMMENTO AD UN TESTO DI LUCA \\ DIOTALLEVI 9}

SANCHIS, Pierre. Desencanto e formas contemporâneas do religioso. In: Ciencias Sociales y Religión. Porto Alegre, ano 3, 2000, p. 27-44.

\section{Sites consultados (em data 8-9/11/2014)}

www.wikipedia.org/wiki/Islam_en_France

jeanbauberotlaicite.blogspirit.com

www.la-croix.com/Debats/Opinions/Debats/Jean-Bauberot

$\underline{\text { www.education.gouv.fr }}$ 